\title{
Biomimetic and Functioning Artificial Tissues - Mastering Irrigation, Nourishment, Microfluidics and Nerve Networks to Keep the Cells Alive
}

\author{
Edilson Gomes de Lima \\ IENN - Institute of Engineering in Nanoscience and Nanotechnology, Australia
}

Copyright $\mathrm{C} 2018$ by authors, all rights reserved. Authors agree that this article remains permanently open access under the terms of the Creative Commons Attribution License 4.0 International License

\begin{abstract}
This paper attempts to bring the view from mechanical engineering from complex industrial processes to tissue engineering. With the intention of bringing this contribution to those involved in tissue engineering, this study sought to be meticulous and to present the key points that can provide significant results. Among one of the contributions is the discussion on the subject, from an engineering point of view, and suggestions such as the generation of engineering diagrams, mapping and detailing each part of the study of the human body. And these engineering diagrams identified by code I.D. each component of the tissues call a datasheet. In this multidisciplinary document with the maximum information and characterization of each component of human tissues, all these information can be used to feed machines and better understand the compounds of the human body in near future. Providing a complete documentation and a nanotopography mapping of the tissues with nanotechnological information and dimensions to the tissues, it is not just a matter of automating tissue engineering, but moreover, understanding the entire biological process system globally. Just as it is done in engineering diagrams of process industries, in analytical engineering, but focused in bioartificial cellular matrix. With this local and global comprehension in tissues, new steps for the field and new challenges can be taken.
\end{abstract}

Keywords Nanoscaffolds, Nanotopography, Tissue Engineering, Mechanical Engineering

"An organ is a complex biologic reactor for interaction between gases, fluids and chemical, organic synthesis processes, not so different from what we have in some industrial physical-chemical complex processes, like ammonium production manufacture, so, analytical mechanical engineering has good tools in this approach".

\section{Introduction}

This research paper focuses on the mechanical engineering point of view. For converting this knowledge in theoretical and practical uses in biosciences, to complex systems in engineering tissue, using the expertise of industrial process engineering as a model, the idea is to attach tissue engineering to the techniques used in large industrial facilities of physical, chemical and biological processes. In industrial plants there are equipment, tanks for reactions, pumps, pipes, several valves and complex instrumentation, all linked to a global control system. Through the customization and design of process this expertise can bring a new way for nanomedicine, generate new techniques, with new tools and allow a new chapter in tissue engineering and in treatments. There is already a large global community involved in the nanomedicine subject, artificial tissues/organs, biosensors, nanotopography [1], scaffolds, methodology, tissue engineering and addition manufacture. The approach and project presented throughout this paper are based on three fundamental complementary projects on tissue engineering:

I. The HGP human genome project.

II. The $3 \mathrm{C} / \mathrm{Hi}-\mathrm{C}$ chromosome conformation capture techniques that are becoming a major design identifying the function of each gene [2], [5] component in the 20.000-strand DNA.

III. The visible human project, conducted by the U.S. national library of medicine.

Also, it's going to be pointed throughout this paper that the tissue engineering will have better results with the union of these three projects, and with the addition of the point of view and techniques of industrial engineering processes, contributing to a future high accurate standardization and customization in works with biology. In this paper there is special attention to technologies that generate patterns in the issue of irrigation, nerve tissue and 
energy networks. Therefore, prioritizing the irrigation mapping by nanotopography and tissue energy is what will make the artificial tissue industry take off. Tissue engineering involves much more than just the deposition of cells by CAD/ CAM systems; there is a whole biological architecture to consider. For example, in the mechanical-chemical refinery process industries, there is the Haber-Bosch process [4], with the full complexity and engineering diagram. It all involves the need for complex molecular biological synthesis, bioreactions, for local-global control and for all the instrumentation involved. And so, we can compare industrial pipes, valves and instrumentation with irrigation veins, biological transport flows and instruments as nerve endings. The objectives are different, but the same can be the means of expertise the production. At the present time, industrial engineering works with complex flow diagrams to accurately to size the connection among chemical and biological reactors. Pipelines with logic control valves, with instrumentation and high overall control. Industrial engineering includes modelling, engineering analysis of chemical processes, fermentation processes, synthesis, mixing, bioprocessing, control, combined processes, optimization and reaction control, among many other complex processes, typical in reactors. The human organs are biological reactors, with $\mu$ channels and valves, requiring measures and accuracy for their due knowledge and control. Everything which is already performed at large scale by diagram engineering.

\section{Methods}

A plant was selected, a beanstalk, as a model and was sliced with a tissue slicer [22], (as in the human visible project) and then every part of this plant was digitally reassembled. All this is to study the plant's endings and irrigation channels. With 100\% clear crystal paper, a mesh grid was printed, dimensionally controlled and specified for the microscope at its magnification. Soon after, the leaf and the sliced pieces of the vegetable were glued in these pieces of sheets. A long, laborious, though not as complex, work as the human genome project, the aim was to map all the major irrigation channels of the middle-aged vegetable. From the root to the leaves of the plant, it provides a better understanding of all its structure of irrigation, breathing and capillarity. This practical example is a model for what this paper means that needs to be done in the human body for a better global comprehension. With the irrigation system and nervous catalogued and with an I.D. number to call a datasheet. With this knowledge, we could e.g. have a better understanding of the functioning of human body pressure, headaches, uncontrolled pressure, and neurological problems. Also, a better understand of the reason for the loss of connection in nervous systems in the spinal cord. And above all, through this knowledge, we would have an effective means of designing artificial organs with better accuracy. Producing an artificial organ is much more complex than just depositing biological fluid in a scaffold mold, it is necessary to have a good knowledge at the level of the human genome project, the $3 \mathrm{C} / \mathrm{Hi}-\mathrm{C}$ chromosome conformation capture techniques and the human visible project. It is all complementary for the mapping of the whole irrigation and nervous structure of the organs. All united in an engineering diagram, and each part with its I.D. can be seen figure 1. as in the electronic circuit architecture [16] demonstrates.

\section{Microchannels are distributed geometrically in order in biology, as in leaves of trees}

By nanotopography, we can transform the microfluidics branches of tree leafs into engineering diagrams, a model to artificial organs and thus automate the biological production, how is it done by industrial engineering

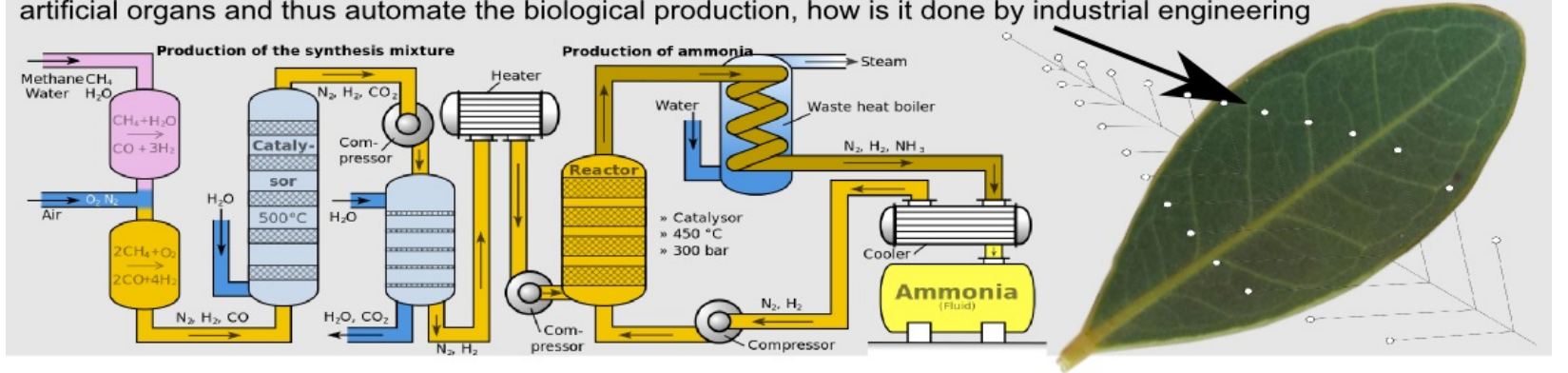

Figure 1. Using nanotopography with engineering diagrams and RF architecture to map the biological geometry
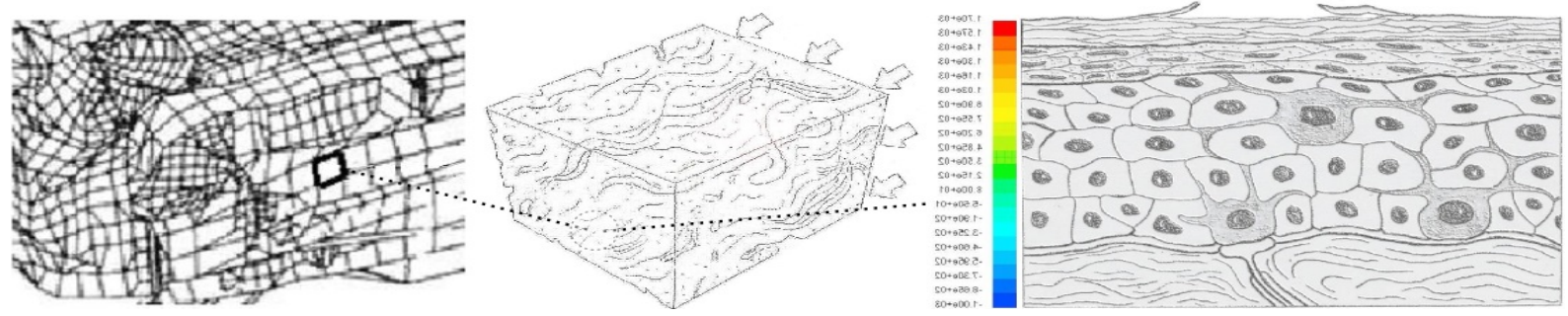

Figure 2. FEM pre-test for low tensile in soft-tissue for structural simulation using Ansys ${ }^{\mathrm{TM}}$ 
Attention to the know-how of the engineering industrial processes for tissue engineering is the key. A combination of knowledge with very effective practices for working with random data, complex systems, organic and biological processes, such as in industrial reactors and engineering process diagrams, contemplating all the microfluidics branching in the tissues. Classifying their biological ramifications, the fluids, and operating conditions. Including all variables and processes, temperature, $\mathrm{pH}$, flow rate, thermodynamics, fluid mechanics, organic reactions and biological processes, it is important to pay attention to the mechanisms of organic reactions [6], and above all, it was used to simulate irrigation lines to measure conditions in elution in $\mu$ vessels as analytical tests by HPLC high-pressure liquid chromatography [7]. It is seen as a reference to the need for complex organic processes of synthesis during the transport of fluids. As result of this study with HPLC an engineering diagram of a plant beanstalk has been developed. So, it's possible to conclude that the human organ must not, necessarily, have the shape or the measure that nature has defined. The organ can have other shape, with some adjustments and accurate calculations in the microfluidic for mimic it, having a perfect copy of the nanotopography [14], but in different and smaller formats, new architecture, but keeping the same process. And in this way, we will finally have artificial organs with complete precision, in commercial formats, defined and projected with the genome project of the irrigation and nervous system. Contemplating all the logic valves involved, going back to ancient studies to understand the circulation of blood and other vital fluids. It is suggested that better control and understanding of the entire network of fluid circulation between cells, tissues and organs is a path to improvement in tissue engineering.

\section{Results}

Designing artificial tissue like a mimicry functional tissue, compatible with the natural is something that goes far beyond what we have today. This starts with materials such as smart hydrogels efficient in some cases, and other materials used for scaffolding. Among the cultivated stem cells, there is still a long way to achieve 3-4D or higher technologies [2] from a point of view beyond geometric. It is a challenge to achieve a perfect mimicry, beyond the topography, surface area with its connections and communication between cells, for functionalization there is still the issue of irrigation. A compatible live tissue involves all communication between the final biologic deposition, with all the human body and the environment; it will have to integrate as a single part. Similar to the parts of the airplanes, they have modern sensors [11], electrical connection with the fairing, with the cockpit and they obey commands, besides the chemical process and realize syntheses in the engines. Thinking about producing artificial organs involves not only geometry, but all these necessary connection, to make the body accept these spare-parts without rejecting it. But for the body to recognize and adapt it takes hundreds of details. A mechanical engineering technique is presented by finite elements methods [15], an important complement to tissue engineering [8] processes. In figure 2 , we can notice an analytical application through the illustration of the technique with FEM in soft tissues using as model a beanstalk phloem structure study.

In the image we can verify a study by finite element non-linear in a piece of tissue, to study the structure and distribution of the cells and its mechanical resistance. Mechanical engineering analytics by features finite element have much to do with a better understanding of mechanics of tissues, including the use of more refined biological materials. FEM is already used in the study of cartilage and bone [9], but there is still much to be done on biological materials, especially in soft tissues. There are good applications for soft tissues, as ducts, microchannels, veins and cells connections will yield results, providing a better understanding of irrigation systems, tissues and fluid interaction and more. It will possible get a perfect distribution in computational systems for tissue printing and a multitude of other applications, as the tissue-engineering scaffold for soft tissues. Under conditions of analysis, calculate the linear elastic displacements, nodal, deformations and tensions in the interaction between the elements, and all mechanics involved in the tissues, aiming the inclusion of the issue of fluidic irrigation, nervous systems, proper hydration and permanent culture of the cells in the tissue, as the integral specific region of the organ, creating an artificial tissue is something that involves several disciplines and every detail needs to be connected. As in computer circuits, it is important to consider where each power line comes from and where it is going, the famous (from/to). The logics involved can be performed by computer scientists, as well as by the engineering techniques in control of complex machines, as it is done with valves controlled by PLC programming. It is important to repeat this point of view, so every thread of life, every vein, and every tangle of the nervous system can receive a TAG / PIN / I.D. number that calls a datasheet. If every connection in the biological body has its precise identification, datasheet, there will be knowledge, where each line of life begins and ends. And with this knowledge, the three-dimensional machines will have the information to be fed. Everything that will be necessary for a future nature mimicking performed, organized and standardized by technological means.

\section{Discussion}

Tissue engineering is a multidisciplinary field, and in this way, a diversified array of skills is required to cover every delicate subject in this discipline with its expertise. 
Tissue engineering [8] is conceptually a science that involves biology, chemistry and physics to generate artificial tissues, aiming the practical purpose of generating artificial compounds, such as skin, organs, healing for burns, cartilage and bones. Facing such a complex subject, it was verified after studies in synthetic tissue that an approach to engineering techniques would be compatible with this field of knowledge. In this way, this paper intends to present some approaches and results achieved. Performing one of the studies in a simple beanstalk, it will be presented throughout this paper. Following the subjects focused on this study:

- Organs are biological reactors with inputs and outputs of fluids of interest.

- The shape and design of organic tissues does not matter if the flow process is ideal.

- Microfluidic and valve control logic in $\mu$ channels irrigation of organic tissues. In comparison with the industrial techniques, such as machine programming techniques, as PLC programmable logic controller.

- Discussion and comparison with the nanotopography design in tissue engineering.

- New approaches for biological nanoscaffold techniques.

- Plants as a model and understanding for the question of networks of $\mu$ channels in organs.

- Engineering expertise as a model in tissue engineering by engineering diagrams.

- Approaches in biometrology and the need for a new anthropometry by nanotopography.

The electronic architecture of bi and three-dimensional integrated circuits has extensive know-how in organizing logical lines, complex connections and high-precision control. The knowledge already used in flow line designs, such as in industrial pipes and valves, presents a great method to be used in the design of artificial tissues and organs. In a tissue or organ each $\mu$ canal is important, each connection between cells, each nerve, vein, control valve is vital, whether in the handling and transport of fluids for irrigation, synthesis in biological reactors or even for energy transport or biochemistry. Therefore, each item needs to be catalogued and receive an identification number with its due datasheet. This is a suggested way for the future generation of biological spare-parts with accuracy, such as the bioelectrical connections between the spine and the brain. In the plant studied, for example, this suggestion is applied on the mapping of the support and reserve phloem, adding an identification number I.D., for each point of transport of water, minerals and organic compounds produced by photosynthesis or captured by the roots and leaves. The mapping method of the irrigation, nutrition and nerve channels can be used on any material: ceramic (teeth and bones), polymers / composites / biological (skin, veins, cartilage), and in any synthetic materials.

\section{a) Stem Cell Culture and Connection Dominium}

A vital point in tissue engineering lies in innovative techniques such as production control and the application of specialized cells to the production of specific tissues. When we visualize an image of a human tissue, organ, fluid or other components by nanotopography, we notice a highly complex surface, with irregular geometry, nothing standardized. In this way, it is not enough to print the tissue with live paint, to reach the natural level. It is a must to understand the mechanism that governs biology, explained in genetic engineering [5] literature in specialized cell culture. Therefore, reaching that complex structure visualized in nanotopography [14], and achieving a satisfactory mimicry, with the same surface area, with vessels, nerves and everything identical to the original, is a challenge. It is necessary to thoroughly understand and have dominium in the specialized cells culture. In figure 3 we can see an exemplification of two complexities that need to be mastered in tissue engineering.

\section{Connecting microchannels, neuronal nerves and Stem cell culture to specialization cells}
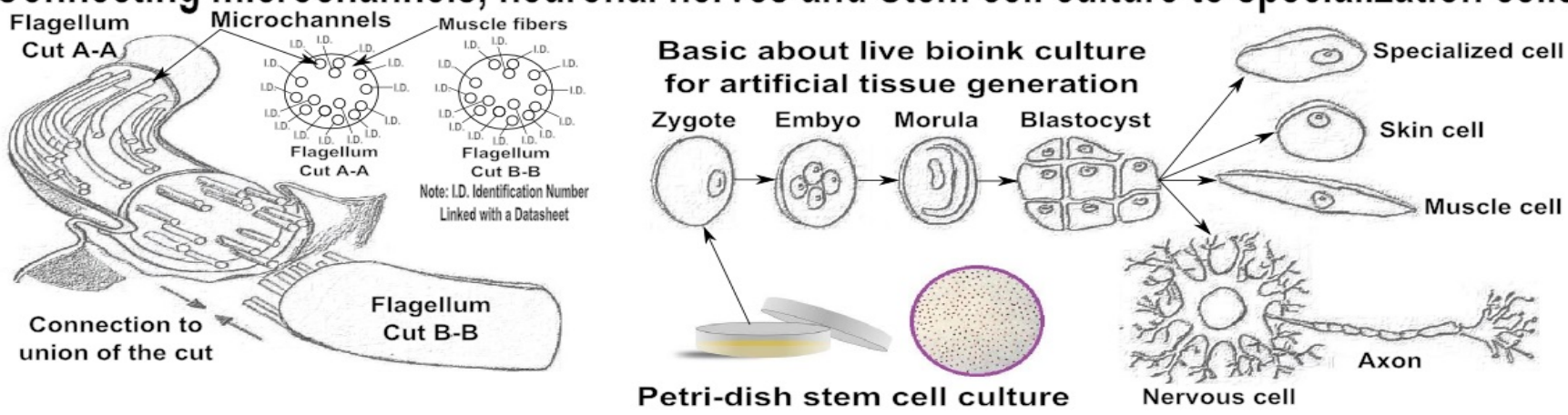

Figure 3. General view on the big challenge of the small connections and the generation of specialized tissues 
As shown, two details about complexity connections in tissues and details about stem cell culture. In this illustration two important biological details are presented. First the complexity in microchannels of the human flagellum mechanism connection, a cut view of the microchannels, and the importance of an I.D. for each one. We can notice details about culture stem-cells with the consequent generation of specialized cells.

\section{b) Nanotopography - As a Tool for Mapping and Catalogue Tissues}

In tissue engineering, there are several important disciplines. However, nanotopography is one of the most relevant. There is not yet a formula for producing organic artificial tissues. However, there are already vital disciplines and tools of high level meaning to this subject. Nanotopography is a term referring to a specific characteristic of an organic or inorganic material, to which the surface area studied is generated on a nanoscopic scale. Nanotopography [12] is not exclusive in tissue engineering; it is also used in micro/ nanoelectronics, in microfluidics, in vegetal biology and especially in biomaterial researches. Besides this there are applications to present in detailed images the nanoscale texture and surface area, for several studies. It is through nanotopography that we understand how nature processes hydrophobicity. Although the focus of this paper is not exclusively on the nanotopography topic, this paper also aims to show the instance for a nanomapping of human tissues, such as artificial tissues, skin, organ, veins, nerves, axons, cellular flagellum, fluids, etc. It is urgent to form a DB of images, geometric details, measurements, materials, as an encyclopedia, at a nanotechnology autopsy level for human biology. And one way to achieve this high-level nanotechnology autopsy of the human body is through nanotopography in conjunction with computer science and engineering methods.

\section{c) Bionanometrology - Information for Feed the New Machines}

As one first step, we need to question how do doctors normally dimensionally collect information from tissues and organs, as well as the possibility of an autopsy. Before starting a project to generate artificial human tissue, it is necessary to define the use, whether for transplantation, laboratory tests, pharmaceutical simulations or search for cures. Usually, the collection of information from a transplant organ begins with some techniques: scan, MRI magnetic resonance imaging, active dynamic thermography, physical-biochemical analysis and others. With these exams, information is generated with precise measurements. From these data, the conversion to digital systems generates geometric models and from these images, the necessary tissue is generated. And with measures, it is possible to calculate the processes, such as the fluid inlet and outlet the same as the original one, for having an accurate artificial organ, with all its bioreactor process, performing its syntheses, mixtures, filtration and other functions correctly. Then check if the internal channel network complies with the correct irrigation, hydration, nutrition for the cells, and sends electrical signals, according to the original biological organs. It is necessary to know the measurements of biological tissues, in the strictest accuracy, for having a possible anthropometry to a standardization of the tissue engineering, with the measure of organic things. In mechanical engineering, we have metrology, which is the science of measuring things with the precision of physics. In chemistry, we have analytical techniques and strategies, such as the characterization materials as a measurement technique. But when we speak about accurately biology measuring, things change. Different symmetries and irregular shapes, even if in the same species, there are evident differences in size, shape, colour, nourishment, and principally the processes. Even if imperceptible, it is enough to change the measurements and avoid a biometrology or bio-anthropometry standardization. Try to find middle ground, with data that need to be treated by mathematics statistics. Find an average that is the closest and compatible with the model that nature produces. Just like in machines, in some cases in biology there may be spare parts, but the process cannot be changed. The tissues and organs present as a bioreactor, performing syntheses, mixtures, filtration, and control conditions such as temperature, flow regulation and organic reactions. Therefore, the synthetics must obey the natural process of the tissues. Similar to how industries regulate the reaction processes, in the case of ammonia production. And by nanotopography we can evidence this mapping, or as a new and complete revolutionary autopsy of the human body by approximations. It's important have a reference measurement of human body parts by $\sim 10^{-9}$ patterns. The measure of the simplest parts in a human body is a big challenge. With measure, bio-machines can be created, and so, new scientific approaches. And a human body biological engineering diagram along with nanotechnology in combination with computational logic. The tissue engineering in relation to organs is in keeping the process adequate to what the complete body system needs.

\section{d) d) The Artificial Organ - From Biometrology for a Question of New Design}

There isn't any magic for cells to become what we want, simply by injecting them into a mold or scaffold [10], with a biological iPSCs flow printer. Even using stems cells, an understanding and general mastery of the subject is still necessary, starting from the beginning, from biology to mechanical and digital logic. For this, a perfectly mimic of the biological surface area by scanning all human tissues by nanotopography technique to form a data base for future 
datasheets is necessary. It does not have a single dimension in biology, precise and standardized. The work with biology is different from the work with solid materials. We still don't have a standard biometrology. In biology, we have much more details. Each person has a different organism biotype adapted to their age process and their organs with specific sizes and shapes. Here we are able to see the need for statistical calculations that seek an average between measures of organs (physical size and data of processes) of people with varied ages. One way to try to standardize the size of the organs would be to measure the most varied models, taking the lungs as an example, seeing that doctors measure the size of 100 to 1000 lungs of people aged 8 to 80 years. These measurements take a table with dimensional, accurate mean passive for interpolation. Perhaps something will be known about the enormous dimensional variation between biological parts. Even so, we must face these dimensional and processes measures, similar to a complete engineering specification to create datasheet as in the electronics. In this way, measures or process data involve many variables, involving thousands of variables, such as the air processing capacity, fluids needed to keep the organ working, maximum and average organ capacity to keep the body functioning. All these can be collected for a database, with all details in fluid mechanics and their precise dimensions, volumes, time, temperatures, $\mathrm{pH}$, enthalpy, and other variables. And with this information, it is possible to begin the necessary engineering work. It is not about re-creating a cyborg, but about mimicking nature, for a better understanding of its functioning, and of the dimensional measurement of biological organs, through nanotopography and biometrology. And one of the ways to measuring is by means of MRI and others. Thus, biometrology can be the source of dimensional data to formulate a complete database of the human body parts, by a complex matrix of multidisciplinary work. Including, measures strategies with magnetic resonance technique, electronic apparatus that measures the distance between two points, or even by autopsy, to which the organ is frozen in nitrogen and sliced to be photographed and precisely scanned in its natural size, as well as by nanotopography.

\section{e) Biological Mapping and Nanotopography of the $\mu$ fluidics $\mu$ channels in Tissues}

This requires a nanotechnological biological anatomy strategy. This is more than a nanotopography of the irrigation tissues of the human body. But also, the mapping of the nervous system, and the smaller details, just perceptible by nanotechnological approaches. It means the need for a new challenge, by a new anatomical approach for all human body by nanotechnology. There may be people who can point that there is already an anatomical mapping of the irrigation system nourishment and the entire nervous system of the human body, but actually not making it more visible with high accuracy and with the approach and engineering techniques as proposed in this paper. This knowledge is more streamlined to the maximum people. Even by examples with electronic, demonstrating each vein, each nerve, from where it begins and where it ends. This study's propose is to present each link, what it does, what its importance is and the consequences of its lack. Much has been done and genetic mapping [13] is being studied by bioengineering with $3 \mathrm{C} / \mathrm{Hi}-\mathrm{C}$ for mapping DNA including the HGP human genome project. Nevertheless, now a general mapping of the microfluidic system of tissue irrigation is included. In figure 4, we can see a reference about what we discuss here. Exemplified in a vegetal, there is what we need to do in the human body, to get all irrigation and nervous system to insert an I.D., inserting all data necessary information.

\section{Details of a beanstalk study for the engineering of irrigation microchannels}

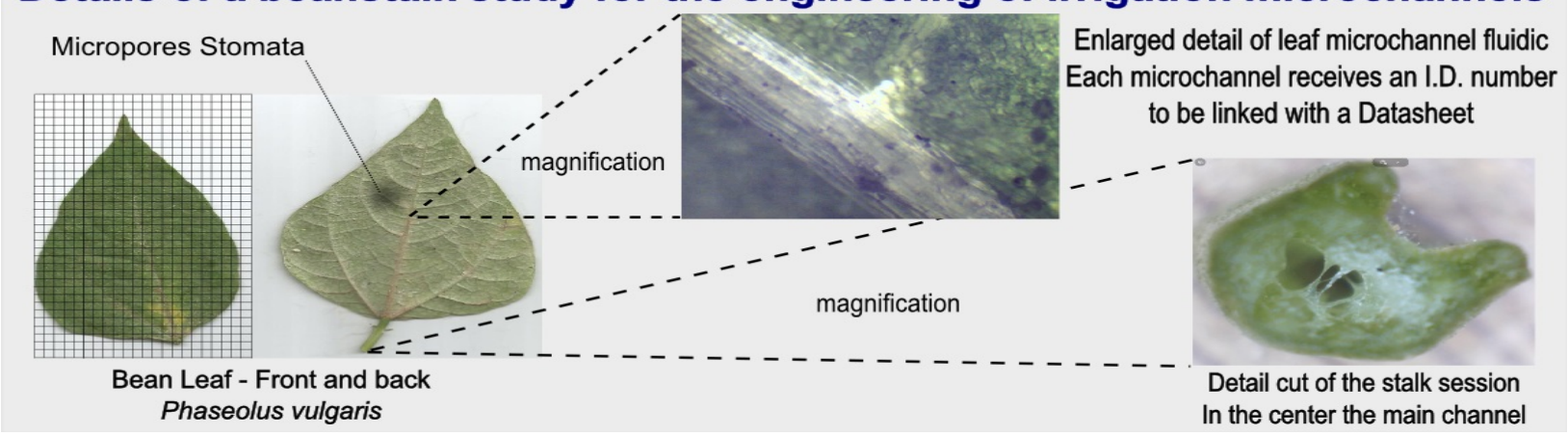

Figure 4. Method used in this study, using a tissue slicer for mapping the $\mu$ channels in a beanstalk Phaseolus vulgaris 


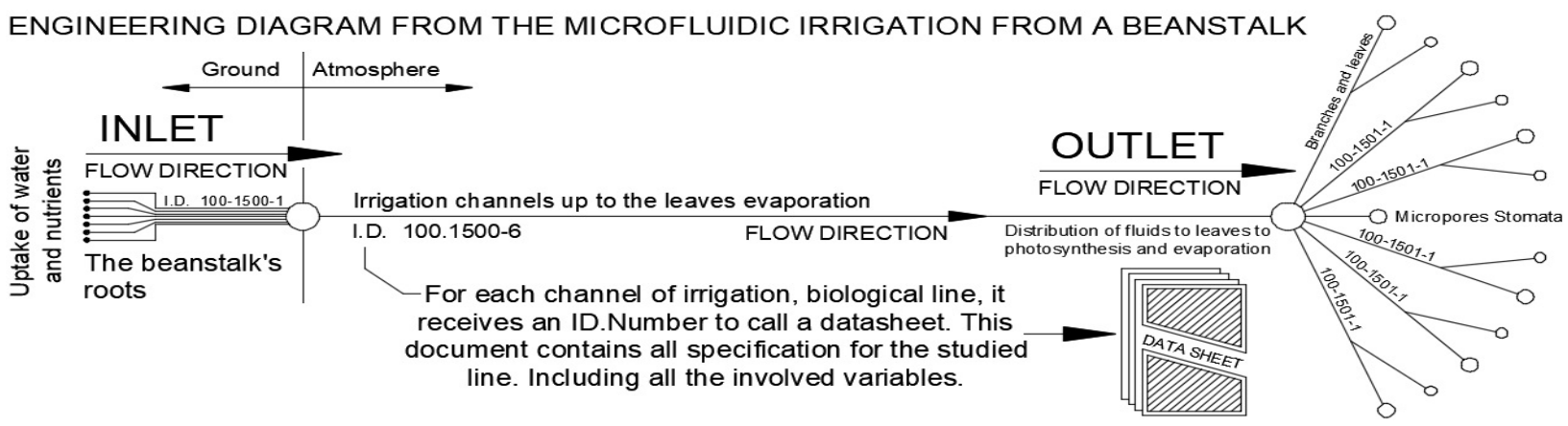

Figure 5. Result suggested of the tissue slicer assembled in an engineering diagram with I.D. for each $\mu$ channel

This study exemplifies the idea behind the science (the visible human project), Which consists of freezing a human body in nitrogen, slicing it into thousands of slices, scanning the parts and then being digitally mounted. In the case of this paper study, the focus is on details of the vascularization of a beanstalk. By this simple case, a new nano-autopsy of human tissues is proposed, through nanotopography, of all human tissues. With special attention to vascularization, irrigation channels, nutrition, nervous and other electrical and biochemical channels of communication in the tissues and organs. With such a wealth of information increasingly accurate computer-aided design machines will be able to deposit the biomaterial, e.g. by electrospinning or even electronically, being a gain for engineering tissue fundamentally. The expected result of this study is the construction of an engineering diagram for an overall understanding of tissue flow, especially the low irrigation, of very low pressure, representing each fluid transport channel in the phloem of the beanstalk. It also aims to study each nerve ending, and other detail contemplated in the diagram. However, each irrigation channel contemplating the origin and the destine or (from/to), where it starts to where it ends, with the addition of an I.D. identification number, and by this number is called a datasheet. In this document the specification, characterization will be a detailed scientific study of each irrigation channel, nerve ending, or specific point studied of the plant. Only with a highly precise engineering can we bring the machines to the correct information for a future and effective production of artificial tissues and organs, as exemplified in the figure 5. But first, we must make the machines understand the whole biological structure. And we can start from the irrigation channels to the structures of complex biological mechanisms. Similar to the case of the flagellar structure, which very much resembles a nanorobot structure, nanotechnology is fundamental. We can see a model for a bidimensional engineering diagram for the irrigation channels of a beanstalk. It is an exemplification to be applied to artificial tissues and organs, for feeding the machines with this information, in high precision by some computer-aided design systems. In the case of the human body, this technique can be used to map the bio-lines and veins, the nervous system, the network of neurons and axons of the brain. Also, other irrigation systems and the structure of the cells, including DNA and thus get all engineering information and most complex variables of the human body, numbering and cataloguing, each microchannel. Some important processes information in datasheets include the diameter of $\mu$ channels, flow per second, kind of flow, characterization of liquids, specification of biomaterials, data about cells, kind of culture cells used, and much more. Changing a three-dimensional structure to a two-dimensional one is ideal for more elaborate studies in human biology. Knowing how to change a complex three-dimensional structural matrix of a human tissue or organ to a two-dimensional plane will allow advanced calculations and studies.

\section{f) Different Visions of Different Disciplines to Aim High at Common Goals}

A curious case happened once in an office, where there were some workers and a nurse working there. A colleague cut his hand in a work accident, after staining the blood; he applied alcohol in the cut. And the nurse scolded him, warning that he could affect a nerve with the alcohol and cause some possible damage, something perhaps improbable. The question seemed to be very subjective and without a scientific reason, even acknowledging that it made a lot of sense. But the question was: what nerves could suffer some damage by direct contact with anhydrous alcohol on the exposed wound? What tissues and what parts could be affected by alcohol contact, or simply there is no problem? By formulating questions like this we verify the importance of a better understanding, through cataloguing, identification and datasheet of each nerve, vein or each minimum connection, where it comes from and where it goes. The engineer needs to have an engineering diagram, to know what each wire, each fluid line performs the (from/to), and each one with an I.D. number and a datasheet. So, the final question is whether it is time to start building datasheets for the human body or not. And so, design an engineering diagram, full of $\mu$ channels, valves, and circuits controlled by various sequences of instrumentation. Consequently, for each line a TAG/PIN/I.D., is received, which is a well-identified 
numbering to call a datasheets similar, how it is done in process engineering in industrial plants and complex machines. And through this datasheet, it will be possible to identify exactly what each channel carries, with technical details, measurements, of flow, pressure, temperature, nutrients, and especially of their connections along the route, where it comes from and where it goes. With this knowledge an engineer can calculate operating conditions, pressure, temperature, set the fluid to be transported, and seek to achieve the best local and global income. Maybe to a doctor, this may seem irrelevant, or perhaps useless, but this view of engineering in medicine has many endless applications, e.g., better comprehension on the high pressure in the brain, or to feed the new machines with accurate information by datasheets, with accurate data of smaller part of the human body. With this accurate information, it would be possible to think of new shaper and designs of tissues, while at the same time in advanced studies for general tissues as organs. So, does a biological organ really need to have the model or shape we know it, or can we think of new formats, through studies in engineering diagrams? Take a note about the importance of the human visible project, figure 6 . To a question like that, we must include the vision of a designer of electronic circuits or piping valves as example. To which, knowledgeable architects of electronic circuits know the meaning of logics to control electricity in $\mathrm{eV}$. For those who are aware, you have already noticed that we are moving towards a biological cyborg project, but for tissue engineering using.

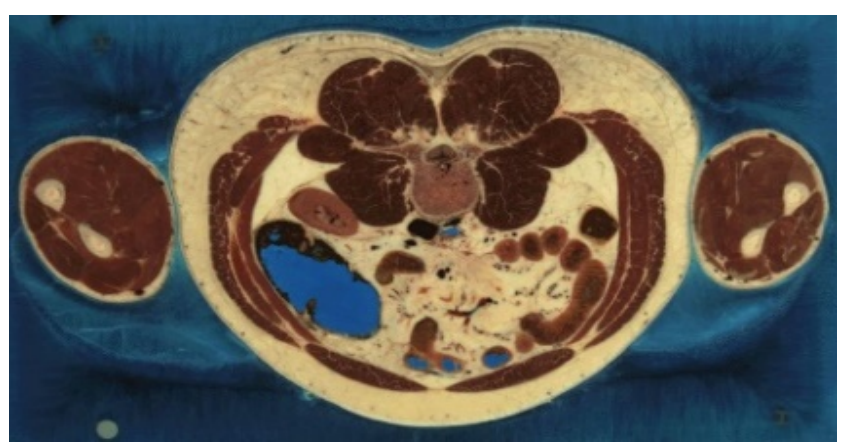

Figure 6. The human visible project - slice of human part. "The visible human project would not be underutilized"?

\section{g) Mechanical Engineering Diagram for Tissues and to Organs}

Taking as reference the knowledge of mechanical engineering from the point of view of the process industry, especially those involving the largely well-defined piping by the ASMETM standards, valves, instrumentation, controllers, complex logic systems, we can approach the production of artificial tissues, as well as new tools for comprehension of irrigation and electrical circuits of the nervous system and other connections. Because it is important that biologic tissue production becomes a discipline for engineering and thus attracting more minds to this science. In short, what is suggested here is the practical and customary use of analytical mechanical/chemical engineering to simplify tissue engineering, maybe for future standardizations, as we see in ASMETM and ASTM ${ }^{\mathrm{TM}}$ for mechanical. Before thinking about tissue/organ production, we need to think in nanoscale. Recall that the DNA is on the $10^{-9}$ scale. Therefore, before thinking about organic printing, it is necessary to know, list and catalogue in depth the smallest parts of biology. In near future, if turn possible connect the stem from one cut beanstalk to another accurately, probably will be possible to connect one arm to another, each time with more accuracy, considering the minimum connections involved. An impertinent issue raised by this article consists of after decapitation observations on various beings, including, insects, warm-blooded animals, and humans, survival depends on several factors, such as vein vessel connection architecture, like cockroaches that survive for many hours after decapitation. This phenomenon is directly linked to a rapid coagulation system and to an independent branched network design, vessels and nerve endings in a very intelligent architecture. The advanced process engineering techniques presents and agrees that the size and format of an organ do not matter if it maintains its proper function and process that the body system needs. It is possible with the engineering techniques by the process engineering diagrams and a bi-three-dimensional design of integrated circuits for biological architectures. A study on energy gain and yield in the process industry involves a complete engineering diagram, considering each interconnection and its physical, chemical and biological process variables. With the control of the processes, it will be viable to generate new formats for the organs, figure 7. In industrial equipment, it is possible to control the process precisely as needed. As the age progresses, each organ adapts to the size, fluid flow and general process. The challenge is finding a middle-term calculation for an artificial organ that supra the process of a person independent of his age, being it from eight to eighty years, that the process can be adjusted. A new three-dimensional architecture based on the themes presented in this paper, can lead to new technologies to produce artificial organs. Fulfilling their functions, regardless of size and shape, so is it possible to change the shape and keep the inlet and outlet process the same? The answer is positive because there's already been artificial organs use in several cases. What this paper presents is a new approach by engineering to improve the quality in the mimicry of organs, to the point where the product is as perfect as the natural ones. Paying attention to the minimum details, to the connections, to the terminations, where each thread of life begins and ends, in addition to accurately understanding what their functions are locally and in the whole. Then, concentrating on inputs, similar to when someone is building a wall, selecting and knowing the materials is essential, see table 1. In artificial tissues, 
things are much more than understanding cell culture, it takes engineering work, through an engineering diagram of all connections, each with its I.D. and perfect identification by a datasheet. With such information in nanometre level, everything will be easier. The tissue printing machines will have concrete information to work with.

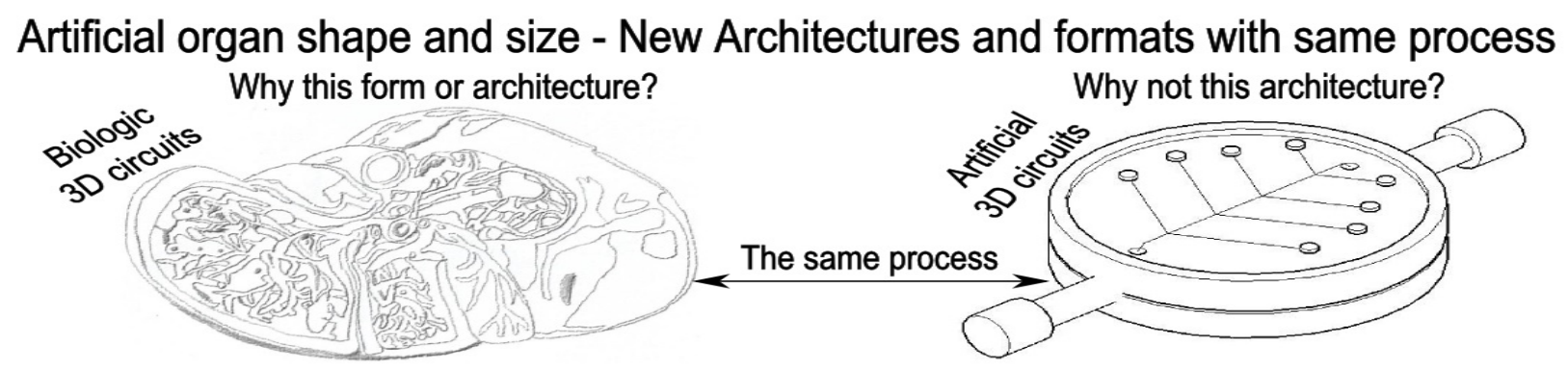

Figure 7. In an artificial organ the size and geometry does not matter, if the same inlet and outlet flow process in maintained

Table 1. Theoretical presentation of each discipline by each group of knowledge and control

Do the organs need to maintain their original shape? In a time that we have new technologies, viable new materials and designs. Everything that exists is based on information organizing matter, as in biology.

How does a layman see a heart?

Generally, people have the power to influence. With definition of supports and actions, and in the case of an organ as the heart, they see it as in the image.

How does a designer see a heart?

Paying attention to details, determining technical elements of drawings. The form representation and techniques - CAD / CAM. Formats, size and connections. Technical representation of complex forms.

How does a doctor see a heart?

Through knowledge of the autopsy terms, knowledge of the connections and interconnections between the heart and the whole. Knowledge of health and disease spectrum. Knowledge of the ideal operating conditions.

How does a computer scientist see a heart?

It is usually seen through computational logics, data organization, intelligence, big-data, data classification, technical reports, organization and classification. Practical means for obtaining results in complex information.

\section{How does an engineer see a heart?}

It is often seen through flowcharts, diagrams, I.D., identification of lines, standardization, accuracy, calculus. Organization of data, information and results in tables and interpolation. Scientific diagrams with analytical data, to develop of complex logical representations. From the point of view of industrial engineering, having a practical technique for working with tissue engineering. A work in part based on the Charles Babbage researches.

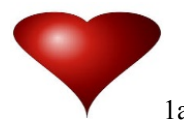

Note: All images 1a to 5a. from Wikipedia Commons

Each professional has a specific knowledge and know-how. Usually each professional only works in their specific area. However, if the compatible knowledge of each discipline is combined with a single objective, innovation will occur. An example is shown in the sequence of images, which focuses on the heart and on its irrigation system, showing that each professional has a different way of seeing a heart. And this paper defends the thesis of joining these competencies with a focus on tissue engineering. But, the objective is presenting the engineering diagram with all the circulatory human system, including neural and microfluidics net. With this engineering diagram it will be possible to mimicry the tissues with high accuracy. 


\section{h) Artificial Organs a Question of Input and Output}

If the process is correct the shape and the size of an artificial organ don't matter at all, if it meets vital biological processes and is able to keep alive and nourished the cells and biological materials that make it up, for due bioreactor process. We consider the tissues here as bioreactors, due to our focus on the irrigation. Similar to tissues, it does not matter whether the organ is fluid, soft or hard, when considering more specifically the tissues, the epithelial, muscular, nervous, connective, bony, cartilaginous or sanguineous systems. If the input and output attend the local and global process, then it's ok. It is vital knowing and defining accuracy, even if approximating to biology. In this study, the size and the biological measurements took place on a beanstalk as a model. We notice that from one plant to another, even being the same species and being cultivated under the same conditions, there are subtle dimensional differences, just like we can tell different human face from the other. So, if a beanstalk does not have the same microfluidic channels, from one plant to another, how could we create an organization, classification and standardization for biology, specifically for biometrology of the tissues? Going deeper on this subject, considering that plants today are genetically modified, and there is a change in their natural structure, the case of transplanting limbs and even the head, is not applied. The case of human head transplantation requires the connection of thousands of these microchannels fluids and nerve endings. If we cut the stem of a beanstalk and transplant it into another, even connecting all phloem, some problems will occur. However, what is defined in this paper is the geomapping of these microchannels, in slices aiming a global understanding, and consequently a future standardization. Soon we will be able to transplant one beanstalk to another with certain success. And if this is possible for a beanstalk, then the head transplant will be better known in this regard. At least, it can help with cell nutrition, or even collaborate with the rejection of transplanted tissues.

\section{i) Scaffold Functionalized - A Theme that Needs to be Understood}

Assembling an artificial tissue or organ is more than mounting cell by cell. It is actually the processes of connecting, linking, interacting and nurturing a full interaction with the biological global system. It also involves nanotechnology techniques such, as bottom-up and tops down. The tissues need irrigation, control, pressure, temperature, nutrition, biosynthesis, similar to a bioreactor. And the materials used to make artificial tissues are another important issue. Cultivated cell culture and antibodies controlling are likely ways to succeed. Nevertheless, we must consider materials such PDMS and so many others to create microchannel models, scaffolds, prototypes, or lab-on-chip $\mu$ channel for tests. The medical oncologists are aware of this and will be vital in this process. By working in partnership with tissue designers, making use of the new nanotechnological biomechanical means. The scaffold could may be in a near future the own irrigation system, including living cells within some forms of a biological scaffold. Through a more advanced point of view, we could say that besides the architectural scaffolds [10] we could include some advanced therapeutic or medicinal process, something to help preserve the body working as an artificial antibody to protect the biologic tissues. We need to define and create the nanomanufacturing process, a pathway leading to a real sophisticated biological manufacturing connected with the IoT internet of things. This process would extend the doctors' reach to computer-aided treatments, to the biomimetic and to the tissues and organs artificial functioning, by mastering the irrigation the microfluidics and nerve networks involved to keep the cells alive.

\section{j) Mechanical Valve Logic from Engineering Diagrams to Tissue Engineering}

The logic valve is a term that defines biomechanical valves in some processes' controlling, like in the flow control of any fluids. For the sake of controlling in the flow of biochemistry, biology and engineering, studying and better understanding the positions and the uses of these biomechanical valves, as done in engineering diagrams are necessary to define the valve and its logical functions. Logically, each valve can control the flow by: directional, separator, intake, loader, leveler, command, shutdown, detour, filler, exhaust, relief, inversion, pressure limiter, start, bypass, pressure control, flow control, turbidity control, overpressure, suction, depressor, brake, magnetic, analytics, regulator, metering and blower. As an example, the heart has the replacement and biological retention valves, and in the body, there are many ways to control fluids, pressure, temperature, $\mathrm{pH}$ and thousands of other physical and chemical variables. The valve logic defines its function and its performance analysing the system in a global way working as an engineering diagram focused on the global process. The total control of fluids in a complex organic system needs logic discipline, such as valve logic. So, after controlling the fluid process, we can start demonstrating how to produce artificial organ, keeping the cells alive. We can also demonstrate the discipline of valve logic, and the technological forms of laboratory customization by artificial organ tests. The customization of laboratories involves several technologies to mitigate and reduce space, time and costs of large laboratories. An example is known by the analytical lab-on-chip, which is a technology that refers to small plaques that can simulate organs, flow channels for unitary operations, microfluidics in general, in the pharmaceutical, physical-chemical, and general analytical tests industry. The analytical lab-on-chip can simulate general biology, chemistry and fluid physics. The organ mimicry study also involves issues concerning the production of cyborgs, as the design of tridimensional circuits [16], and even experiments linked to 
transhumanism. The process of fluid circulation in large living beings, such as whales, humans and elephants, shows a complex matrix of fluids, and mimicking this array of channels is a challenge. One of the direct applications of these new technologies are experiments, and above everything and as important as combating diseases effectively, is the prior identification of potential problem. One of the techniques of identifying cancer beforehand is the use of markers. This use acts as a kind of "hard finger", which denounces that there is something wrong, with the aid of these technologies, such as simulation of blood circulation in microplates, it is possible to perform automated tests. For this, the markers act together in the interaction with specific antibodies, although some antibodies only appear when the disease is running, it is still important to anticipate it. So, this is one of the clues, and for every antibody, there is a specific disease. Therefore, a technology that can anticipate this organism's natural action would be vital for the technology of immense scope.

\section{k) The mechanism Behind biology - Biologic Synthesis of Flagella by Nanotechnology}

In order to absorb the techniques among different disciplines, with a focus on tissue engineering, an interesting approach is the use of nanotechnology techniques to mimic complex points of biology, making use of top-down mechanical and bottom-up nanotechnologies. One of the points of high complexity in biology [18] is in DNA and in the composition of each unit cell in mechanisms such as human flagella, as we can see details in figure 8 . Due to the complexity of the flagellum mechanism, it makes it clear that understanding microfluidics, the capillarity, branched network of blood vessels, and nerve endings is necessary. The mechanism behind flagellum, a growth sequence in the synthesis of the flagellar structure, understanding the mechanism of complex flagellar structures [17] is vital for the development of tissue engineering. The domain of the flagellar structure synthesis including the use of nanotechnology techniques [20] with studies in motor proteins is one of the necessary steps for high-level tissue engineering. And one of the tools for this domain is in bionanotechnology, especially in molecular biology of machines assembling [19]. The presented issue is how to efficiently synthesize the flagellum, through tissue engineering, considering a synthesis for characterization of surface area of complex tissues as a model.

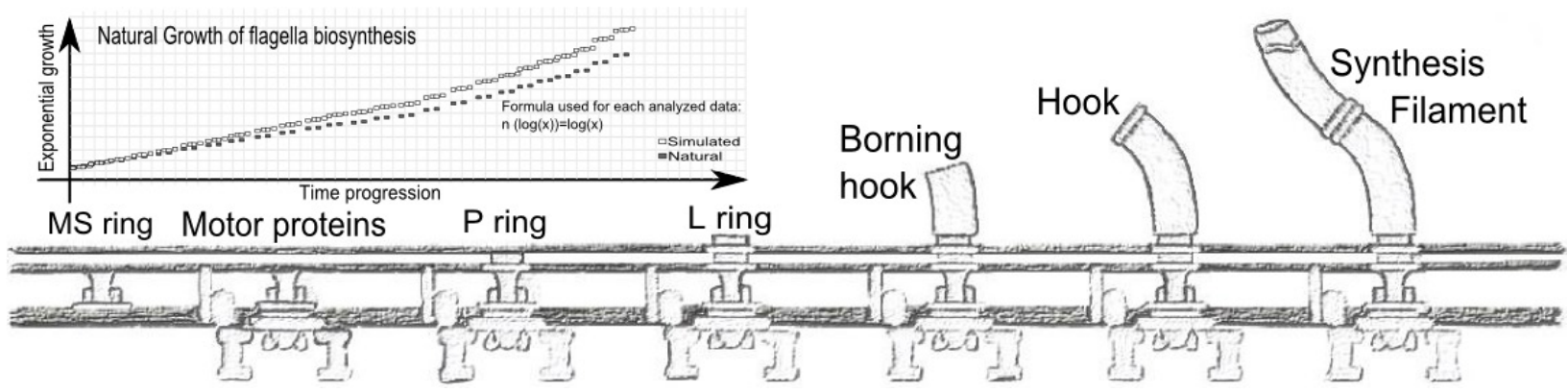

Figure 8. Detailed presentation of a complex biological unit growth as a reference in tissue engineering control, performed by natural growth of flagella biosynthesis timing

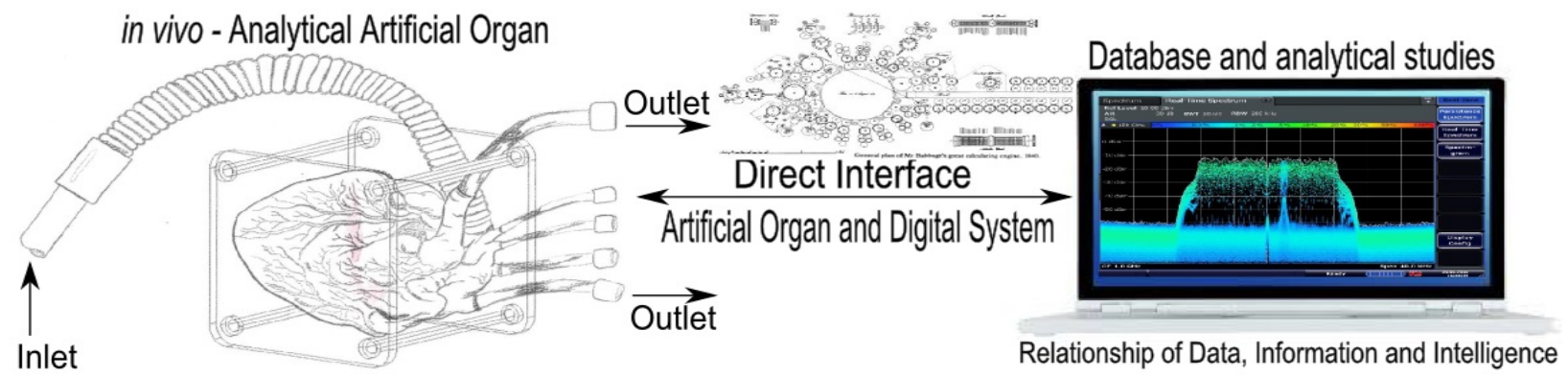

Figure 9. Analytical artificial organ for high-level study in vivo to perform tests on artificial tissues 


\section{Possibilities and Results}

Through this path, we can approach an analytical artificial tissue/organ to real tests, and advancement in tissue engineering to produce analytical equipment for laboratory tests, just like creating an illness in an artificial organ, as a copy of the patient illness, and putting it in a laboratory table for real tests. This would lead to alternatives and no risks treatment studies. Considering an artificial organ with a tumour, or e.g. an artificial heart like in figure 9 , we can note a total interface between organism and digital system. So, the organ as one bioreactor will need fluids flow, as serum, blood, energy and other goods to keep it alive. These connections need to be perfect and previously studied by engineering diagrams. Therefore, it faithfully represents and operates the actual organ, as a copy. An important note by the analytical engineer of the British mechanical Charles Babbage, is to remember the then and now from the microcircuits progress to the actual modern architecture. The demonstration of an artificial organ produced specially to simulate analytical tests, having a real copy of the illness in the patient. It is important to note that this artificial organ or tissue laboratory bench is an analytical instrumentation, biological equipment interconnected to the digital system interface [3]. With this analytical instrumentation that will be possible to apply the concepts proposed in this paper, such as the identification of each vascularization, vein, nerve, cellular set and all tissue mapping by nanotopography of all the biological matrix. This process will be useful for comprehending better the microstructure of micro veins in brains, in a nutshell, applying the concepts of high-level engineering in biology. In design integrated circuit is used in high-level logic and engineering techniques, for the goal is each circuit to receive an I.D. identification number and find a specific datasheet for that circuit line. In the datasheet, there is the complete specification of circuit line, where it starts and ends, with all the details, the diameter, the capacity, the function, and the technical information. All this work involves a gigantic base of analytical control, and as a reference, we have the work by the mechanical engineer Charles Babbage [21], focusing on his analytical precision with an interface in mechanics, and now for biology.

"As soon as an analytical machine exists, it will necessarily guide the future course of science. When a result is sought for your help, the question will be raised in the act. By what calculation path can these results be obtained with the machine in the shortest time?" - Charles Babbage, scientist and mechanical engineer

\section{Outlooks and Proposition}

This paper ends presenting the possible construction of spare-parts human organ and a functionalized tissue for using in analytical practices. It would be an artificial analytical tissue in vivo, fully functioning, mimicking the real tissues. Being an organ, a skin, a bone, a capillary tissue, a branched network of vessels and nerves, fully functionalized. It would be available for the majority of the researchers, doctors, laboratories, universities, pharmacies, and research centers, and also an instrument of analytical studies, in a revolution to the laboratory-on-chip. The artificial, organ-shaped tissues will perfectly simulate the actual, mass-produced one, and will be available for purchase and for use at any research centre or at various tests. Artificial tissues will completely mimic living organs, e.g. a tissue presenting a diseased organ can be used analytically for clinical studies, or even be analyzed by the most diverse disciplines. While they will be analyzing the artificial organ pulsing, in vivo, they will be conducting their research and verifying results, trying to validate their studies. It will also be a new modern biological instrumentation for research. Theoretically, in any laboratory there would be an artificial organ in full operation, simulating the most varied anomalies, diseases and malfunctions for complex studies. A practical case would be to develop an artificial organ based on the biology of mole-mouse stem-cells, which is immune to cancer for advanced studies. It will be a new revolution for in vivo research to which these tissues, organs and biological parts can be connected to computer-systems for collecting information in real time and for controlling its function. Also, it will be possible to have administration and pharmacodynamic testing, invasive testing, on a stand, direct in the target tissue in vivo, having the visualization of the test by computational system, and monitoring the biointerface. With the software connected to the tissue and to a database, it will contain all information of the structure of the organ. This analytical apparatus will read the indicators, the markers, each signal in $(\mathrm{eV})$ electron Volts, each biochemical signal, each interaction between nanoparticles, and all the tissue by biosensors monitoring. Finally, as a challenge, there is too much information that should be added to the engineering diagrams to detail each $\mu$ fluidic circuit of the tissues in all its structure. In search for global standards it will be possible to perform the complete mapping of a hair in comparison to a similar one. Performing all steps: biosynthesis, cataloguing, identification I.D., mapping, engineering diagram and datasheet. And in the future, freeze tissues and organs, slice and map the network of fluids, making use of $\mu$ circuit technology. Moving from three-dimensional to two-dimensional biological structure, with this mapping and the engineering diagrams, we will have more information on the flow of nutrition, circulation, electrical communication networks and operation data, towards a complete work of the low circulation of the feet, passing through the spinal cord to the brain. Basing this study on the future perspective of a better reach in the research focused on the mimicry and functionalization of artificial 
tissues, focusing on the structure of irrigation, nutrition, microfluidics and nerve networks needed to keep the cells alive.

\section{Acknowledgements}

Financial \& competing for interest disclosure - The author has no relevant affiliation or financial involvement with any organization or entity with a financial interest in or financial conflict with the subject matter or materials discussed in the manuscript, including employment, consultancies, honoraria, stock ownership or options, expert testimony, grants or patents received, pending or royalties. No writing assistance was done in the production of this manuscript. And the indicated institute is totally no profit, so, this manuscript has the intention of collaborating with the spread of knowledge.

\section{REFERENCES}

[1] Alexander R. Votruba, et. al. Nanotechnology in Drug Delivery and Tissue Engineering: From Discovery to Applications - C2010 - DOI: 10.1021/nl102184c

[2] Gavrilov A. et. al. Chromosome conformation capture (from 3C to 5C) and its CHIP-based modification - C2009 DOI:10.1007/978-1-60327-414-2_12

[3] I. Scott MacKenzie - Human-Computer Interaction: An Empirical Research Perspective 1st Edition - C2013.

[4] Jayant M Modak - Haber Process for Ammonia Synthesis Indian Institute of Science. C2002.

[5] Cynthia E. Dunbar, et. al. Gene therapy comes of age http://dx.doi.org/10.1126/science.aan4672

[6] Robert B. Grossman - The Art of Writing Reasonable Organic Reaction Mechanisms - Springer, 2nd - C2007

[7] Tim Wehr, Robert L. Cunico, Karen M. Gooding - Basic HPLC and CE of Biomolecules - C1998

[8] Bernhard O. Palsson, Sangeeta N. Bhatia et. al. Tissue
Engineering C2016

[9] Feng Zhao, Jian Wang, Hongjuan Guo, Shaojun Liu, and Wei He - The Effects of Surface Properties of Nanostructured Bone Repair Materials on Their Performances - Hindawi C2015

[10] Peter X. Ma, Jennifer Elisseeff - Scaffolding in Tissue Engineering - CRC Press C2005

[11] Vijay K. Varadan, LinFeng Chen, Jining Xie Nanomedicine: Design and Applications of Magnetic Nanomaterials, Nanosensors and Nanosystems - Wiley $\mathrm{C} 2008$

[12] J. McMurray, et. al. Nanotopographical Control of Stem Cell Differentiation - Journal of Tissue Engineering C2010 doi:10.4061/2010/120623

[13] Ming-Cheng Luo, Karin R. Deal Armond, et. al. - Optical Nano-mapping and Analysis of Plant Genomes - Humana Press, New York, NY - C2016

[14] Kriparamanan R, Aswath P, Zhou A, Tang L, Nguyen KT Nanotopography: cellular responses to nanostructured materials - C2016

[15] Dr.Javier Bonet, Dr. Richard D. Wood - Nonlinear Continuum Mechanics for Finite Element Analysis Cambridge Press - C2008

[16] Baltus, P. G. M. (2004). Minimum power design of RF front ends Eindhoven: Technische Universiteit Eindhoven - DOI: $10.6100 /$ IR580521

[17] Ken F. Jarrell et. al. Prokaryotic motility structures Microbiology (2003), 149, 295-304

[18] Larry Snyder, Joseph E. Peters, Tina M. Henkin, Wendy Champness - Molecular Genetics of Bacteria - C2013.

[19] Steven, Baumeister, Johnson, Perham, et. al. - Molecular Biology of Assemblies and Machines - CRCPress C2016

[20] Tuan Vo-Dinh - Nanotechnology in Biology and Medicine: Methods, Devices, and Applications - C2007.

[21] Charles Babbage - Reflections on the Decline of Science in England - Dodo Press C2006

[22] Martina Zimmermann, et. al. Improved reproducibility in preparing precision-cut liver tissue slices - C2009 DOI:10.1007/s10616-009-9246-4. 